\title{
Propiedades psicométricas: Escala de asertividad en escolares peruanos
}

\author{
Psychometric properties: Assertiveness scale in peruvian schoolchildren
}

\author{
Fernando Joel Rosario Quiroz ${ }^{1}$ \\ Universidad de Lima
}
César Raúl Manrique Tapia ${ }^{2}$, Erika Roxana Estrada Alomía ${ }^{3}$
Universidad César Vallejo
Miguel Ángel Misare Condori ${ }^{4}$
Essalud

Alex Grajeda Montalvo ${ }^{5}$

Universidad Nacional Mayor de San Marcos

Juan Walter Pomahuacre Carhuayal ${ }^{6}$

Instituto de Investigación, Capacitación y Desarrollo Psicosocial y Educativo

Recibido: $16-02-20$

Aceptado: $12-06-20$

\section{Resumen}

La presente investigación tuvo como objetivo determinar las propiedades psicométricas de la escala de asertividad ADCA 1, el tipo de investigación es instrumental; se trabajó con una muestra de 730 individuos con edades entre 13 a 16 a más años. Se utilizó un muestreo no probabilístico por juicios. Los resultados indican que la escala de Auto-Asertividad en su propuesta original no presenta evidencias de validez de constructo $\left(\mathrm{X}^{2} / \mathrm{gl}=2.648\right.$; $\mathrm{GFI}=0.883$; $\mathrm{CFI}=0.807$; RMSEA $=0.048$ y SRMR $=0.0532$ ); después de analizar los ítems se trabajó un análisis exploratorio con una rotación oblicua obteniéndose 5 factores que explican el

\footnotetext{
${ }^{1}$ Magíster, Docente Universidad de Lima. Autor de correspondencia. E-mail: frosario@ulima.edu.pe ORCID: https://orcid.org/0000-0001-5839-467X

${ }^{2}$ Magíster, Integrante del Instituto de Investigación de la Escuela de Psicología - Universidad César Vallejo. E-mail: cmanriquet@ucv.edu.pe. ORCID: https://orcid.org/0000-0002-6096-1482

${ }^{3}$ Licenciada, Docente de la Escuela de Psicología, Universidad César Vallejo Lima. E-mail: eestradaa2@ucv.edu.pe ORCID: https://orcid.org/0000-0001-5916-1034

${ }^{4}$ Licenciado, Psicólogo de Essalud-Adulto mayor - CAM. E-mail: miguelmisare@gmail.com ORCID: https://orcid.org/0000-0002-4749-1260

${ }^{5}$ Doctor, Docente Pre y Posgrado Universidad Nacional Mayor de San Marcos. Lima, Perú. E-mail: agrajedam@unmsm.edu.pe. ORCID: https://orcid.org/0000-0001-5972-2639

${ }^{6}$ Magíster, Instituto de Investigación, Capacitación y Desarrollo Psicosocial y Educativo (PSYCOPERU), Lima, Perú. E-mail: walter.pomahuacre@gmail.com. ORCID: https://orcid.org/0000-0002-6769-6706
}

(C) Los autores. Este artículo es publicado por la Revista de Investigación en Psicología de la Facultad de Psicología, Universidad Nacional Mayor de San Marcos. Este es un artículo de acceso abierto, distribuido bajo los términos de la licencia Creative Commons Atribucion - No Comercia_Compartir Igual 4.0 Internacional. (http://creativecommons.org/licenses/by-nc-sa/4.0/) que permite el uso no comercial, distribución y reproducción en cualquier medio, siempre que la obra original sea debidamente citada. 
$43.602 \%$ del constructo. El índice de ajuste de la propuesta de 5 factores presenta evidencias de validez de constructo $\left(\mathrm{X}^{2} / \mathrm{gl}=2,225 ; \mathrm{GFI}=0.936\right.$; $\mathrm{CFI}=0.900$; $\mathrm{RMSEA}=0.041 ; \mathrm{SRMR}=$ 0.0463 ), a nivel general la escala con 25 ítems presenta un $\alpha_{\text {ordinal }}=0.902$ y u $\Omega=0.902$. En conclusión, la propuesta de 25 ítems presenta evidencias de confiabilidad y validez.

Palabras clave: Asertividad; adolescentes; comportamiento social.

\begin{abstract}
The purpose of this research was to determine the psychometric properties of the ADCA 1 assertiveness scale, the type of research is instrumental; we worked with a sample of 730 individuals between the ages of 13 and 16. It was considered a non-probabilistic sample by trials. The results indicate that the Self-Assertiveness scale in its original proposal does not present evidence of construct validity $\left(\mathrm{X}^{2} / \mathrm{gl}=2,648 ; \mathrm{GFI}=0.883 ; \mathrm{CFI}=0.807 ; \mathrm{RMSEA}=0.048\right.$ and SRMR $=0.0532$ ); After analyzing the items, an exploratory analysis with an oblique rotation is sought, obtaining 5 factors that explain $43,602 \%$ of the construct. The adjustment index of the 5-factor proposal presents evidence of construct validity $\left(\mathrm{X}^{2} / \mathrm{gl}=2,225\right.$; GFI $=0.936 ; \mathrm{CFI}=0.900 ; \mathrm{RMSEA}=0.041 ; \mathrm{SRMR}=0.0463)$, a general level the scale with 25 items presents a $\alpha_{\text {ordinal }}=0.902$ and $\mathrm{u} \Omega=0.902$. In conclusion, the 25 -item proposal presents evidence of reliability and validity.
\end{abstract}

Keywords: Assertiveness; adolescents; social behavior.

En el mundo, desde un enfoque tradicional, se valoran aspectos técnicos para incrementar la productividad a nivel de cualquier sistema que brinde algún servicio, sin embargo, en la actualidad, se han generado cambios al respecto y la valoración de las denominadas habilidades blandas o softskills es determinante, pues están vinculadas a "un conjunto de rasgos de personalidad, habilidades sociales, comunicación, lenguaje, hábitos personales... los cuales permiten a una persona interactuar efectivamente" (Roca, 2015, p.50), sobre las habilidades duras o hardskills que se relacionan con las habilidades relacionadas con el conocimiento. Dentro de esta línea de habilidades blandas encontramos a la asertividad definida como aquella "clase de conductas sociales" que indican el respeto que se tiene uno mismo y a los demás de manera igualitaria, sobre todo con los que se interactúa (García y Magaz, 2011, p. 12), a la vez, indican García y Magaz (2000) que la asertividad es un constructo, el cual se divide en dos constructos, siendo el primero, la autoasertividad, la cual está compuesta por sentimientos sinceros y de la defensa de valores, gustos, preferencias y deseos de manera personal; y segundo, la heteroasertividad, basada en sentimientos sinceros y de la defensa de valores, gustos, preferencias y deseos hacia los demás.

Por otro lado, Güell y Muñoz (2000) citados por Aquilarte et al. (2010) proponen que una persona asertiva expresa lo que siente, piensa o quiere sin lastimar a los demás, es decir, es empático, también impide que sea manipulada, genera mayor espontaneidad en sus interacciones sociales, presenta una más alta autoestima, posee mayor autocontrol emocional y muestra conductas de respeto hacia los demás, aceptándolas o rechazándolas emocionalmente con sutileza. Los 
autores citados refieren que el modelo en el que se basa la conducta asertiva es complejo llevarlo a la vida práctica, debido a que en este proceso de socializar con los demás, se aprende a ser pasivo con respecto a los hechos, teniendo también como formas de aprendizaje el medio social, los medios de comunicación y una educación basada en generar competitividad, creando así una conducta agresiva. Por lo cual, comportarse asertivamente tiene que ver con actuar de manera distinta o contraria a los modelos que en la sociedad se practican. Dentro de esta lógica, es importante explorar como estudió la asertividad Morán y Olaz (2014) quienes realizaron un análisis bibliométrico en América Latina de los instrumentos que evalúan las habilidades sociales y constructos similares como la asertividad, competencia social y autoeficacia, encontrando que los picos donde se realizó mayor cantidad de estos constructos fueron en el 2009 y 2003, además, se encontró que el 59.5\% realizó estudios de habilidades sociales, $18.9 \%$ competencia social y asertividad y un $2.7 \%$ de autoeficacia; en la misma investigación se hace mención que el $51.4 \%$ trabajó con niños, el $18.9 \%$ con universitarios y el $10.8 \%$ con adolescentes en edad escolar lo cual muestra un déficit con respecto a la medición de la asertividad en estudiantes secundarios, haciendo necesaria la realización de investigaciones con este grupo humano.

De otro lado, Inglés, Méndez e Hidalgo (2000) listaron los siguientes instrumentos para explorar las dificultades interpersonales en la adolescencia QSDA, SRSBQ, MESSY, ASA, PARS, S-EFF, CCQ, TISS, EA, CAPS Y IPSIUA que evalúan factores tales como ansiedad social, aserción, control del mal genio, malestar emocional, sumisión, agresividad, conducta interpersonal, conducta relacionada con la tarea, habilidades sociales, aserción inapropiada, autosuficiencia, competencia social, participación social, participación en grupo, amistad e intimidad, dar y recibir ayuda, respeto por las reglas, conductas prosociales, conductas antisociales, mantener amigos, conductas problemas entre otros. A nivel local los instrumentos más utilizados para evaluar la asertividad son la escala de asertividad de RATHUS, que evalúa la demostración de disconformidad, manifestación de sentimientos, eficacia, interacción con organizaciones, expresión de opiniones y capacidad de decir no (León, 2009). El Auto-informe de Conducta Asertiva ADCA 1, es otro de los instrumentos con mayor difusión en el país que evalúa la asertividad desde un punto de vista cognitivo, que sistematiza la manera de comportarse socialmente de las personas; Ayvar (2016) utilizó este instrumento en su trabajo: autoestima y asertividad en adolescentes de educación secundaria de un colegio estatal y particular de un sector de Santa Anita, calculando la confiabilidad de la escala con el método de consistencia interna con un alfa de Cronbach de 0.858 , pero no hace referencia a la validez del mimo, siendo por lo tanto, necesario realizar un estudio psicométrico de mayor amplitud que permita brindar a la comunidad científica un parámetro de comparación. La validez es un elemento importante en todo instrumento pues nos demuestra la utilidad que tiene el mismo para medir la variable en cuestión ósea el cumplimiento de su propósito (Grajeda, 
2018, p. 24). Debido a lo indicado, se propuso la siguiente pregunta de investigación: “¿Cuáles son las propiedades psicométricas de la Escala de Asertividad ADCA 1 en un grupo de estudiantes del nivel secundario de instituciones públicas del distrito de Independencia, 2019?".

Para poder llegar a ello se plantearon como objetivos.

General: Describir las propiedades psicométricas de la Escala de Asertividad ADCA 1 en un grupo de estudiantes del nivel secundario de instituciones públicas del distrito de Independencia, 2019

Específicos:

1. Describir la validez estructural de la Escala de Asertividad en estudiantes secundarios del distrito de independencia.

2. Determinar la confiabilidad de la Escala de Asertividad en estudiantes secundarios del distrito de independencia.

3. Calcular las diferencias en las puntuaciones de la Escala de Asertividad en estudiantes secundarios del distrito de independencia en función al sexo.

4. Elaborar la tabla de normas percentilares para de la Escala de Asertividad en estudiantes secundarios del distrito de independencia.

\section{MÉTODO}

Es una investigación de tipo instrumental, tal como lo indican Montero y León (2002), que es cuando aquel trabajo posee como fin el desarrollo, diseño o adaptación de pruebas y aparatos.

La población estudiantil de secundaria de Independencia está compuesta por 12422 alumnos (Ministerio de Educación del Peru, 2019). El número de la muestra se calculó con un 95 por ciento de confianza y un riesgo de error de 3.5 a partir de la fórmula para poblaciones finitas descrita por Abad y Servin (1984). El resultado fue de 730 sujetos, los cuales son estudiantes de básica regular de primero a quinto año de secundaria, con edades entre 13 a 17 años del distrito de Independencia. Se utilizó un muestro no probabilístico por juicios para seleccionar la muestra, según Ñaupas, Mejía, Novoa y Villagomez (2011) que consiste en seleccionar a los sujetos de la muestra según el criterio propio del investigador.

Se usó el Autoinforme de conducta asertiva (ADCA - 1) de E. Manuel García Pérez y Ángela Magaz Lago, cuya administración es de manera tanto individual como colectiva. Dirigido a adolescentes entre 12 - 18 años que identifica el estilo 
cognitivo pasivo, agresivo o asertivo, que regula el comportamiento social de los individuos.

Los aspectos que evalúa la propuesta original del instrumento es: auto-asertividad: grado o nivel de respeto y consideración hacia los sentimientos, ideas y comportamientos propios y hetero-asertividad: grado o nivel de respeto y consideración hacia los sentimientos, ideas y comportamientos ajenos. El sistema de puntuación por cada ítem corresponde a Nunca (4); A veces (3); A menudo (2) y Siempre (1).

La distribución de ítems tiene la siguiente estructura:

\section{Tabla 1}

Derechos asertivos y elementos del ADCA - 1

\begin{tabular}{lcc}
\hline \multicolumn{1}{c}{ Derechos Asertivos Básicos } & $\begin{array}{c}\text { Ítems } \\
\text { Auto - Asertividad }\end{array}$ & $\begin{array}{c}\text { Ítems } \\
\text { Hetero - Asertividad }\end{array}$ \\
\hline $\begin{array}{l}\text { A actuar de modo diferente a como los demás desearían que } \\
\text { actuase }\end{array}$ & 3 & 22,30 \\
A hacer las cosas de manera imperfecta & 7 & 27 \\
A cometer equivocaciones & $5,6,15$ & 29 \\
A pensar de manera propia y diferente & 1 & 23,30 \\
A cambiar de opinión & 8 & 24 \\
A aceptar y rechazar críticas o quejas & 13,14 & 31,35 \\
A decidir la importancia de las cosas & ---- & 34 \\
A no saber algo & 10,16 & 28 \\
A no entender algo & 12 & 21 \\
A formular preguntas & 17 & 26 \\
A hacer peticiones & 18 & 25 \\
A rechazar una petición & 19 & 32 \\
A expresar sentimientos & $2,4,11$ & 33 \\
A elogiar y recibir elogios & 9,20 & ---- \\
\hline
\end{tabular}

Fuente: García y Magaz (2003).

\section{RESULTADOS}

\section{Análisis Factorial Confirmatorio de la Propuesta Original}

En la figura 1 se presentan los índices de ajuste de la propuesta original planteada por los autores del test de auto informe de conducta asertiva ADCA, $\mathrm{X}^{2} / \mathrm{gl}=2.648$; $\mathrm{GFI}=0.883 ; \mathrm{CFI}=0.807$; $\mathrm{RMSEA}=0.048 ; \mathrm{SRMR}=0.0532$, en todos los casos, los índices de ajuste se encuentran por debajo de los estándares establecidos para indicar que el modelo propuesto no mide idóneamente la asertividad (Escobedo, Hernández, Estebané y Martínez, 2016). 


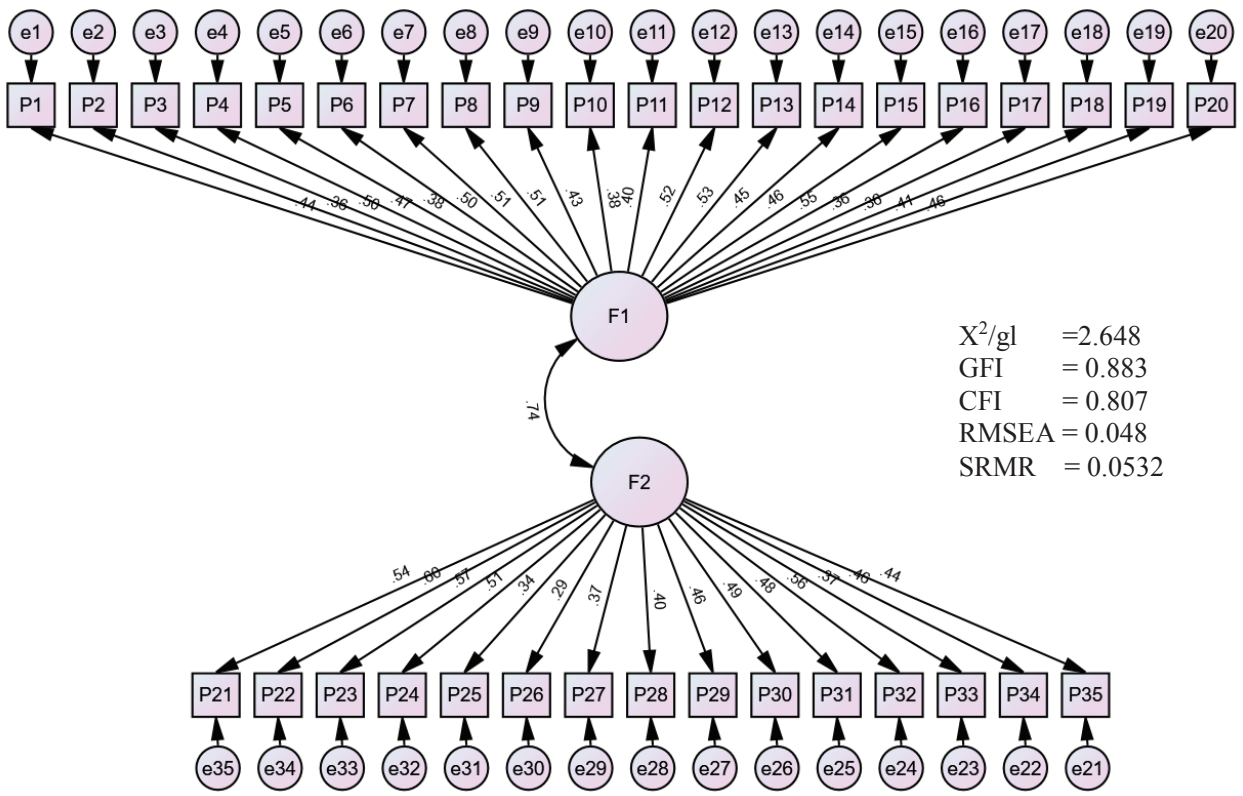

Figura 1. Diagrama de senderos de la propuesta original del Auto informe de conducta asertiva (ADCA - 1)

En la figura 2 se observan los índices de ajuste de la propuesta original planteada por los autores del test de auto informe de conducta asertiva ADCA específicamente para la dimensión autoasertvidad, $\mathrm{X}^{2} / \mathrm{gl}=3.252$; $\mathrm{GFI}=923 ; \mathrm{CFI}=0.840$; RMSEA=0.056; SRMR=0.0499, en todos los casos los índices de ajuste se encuentran por debajo de los estándares establecidos para indicar que el modelo propuesto no mide idóneamente la autoasertividad, solo el GFI y el SRMR presenta índices adecuados. (Escobedo, Hernández, Estebané y Martínez, 2016).

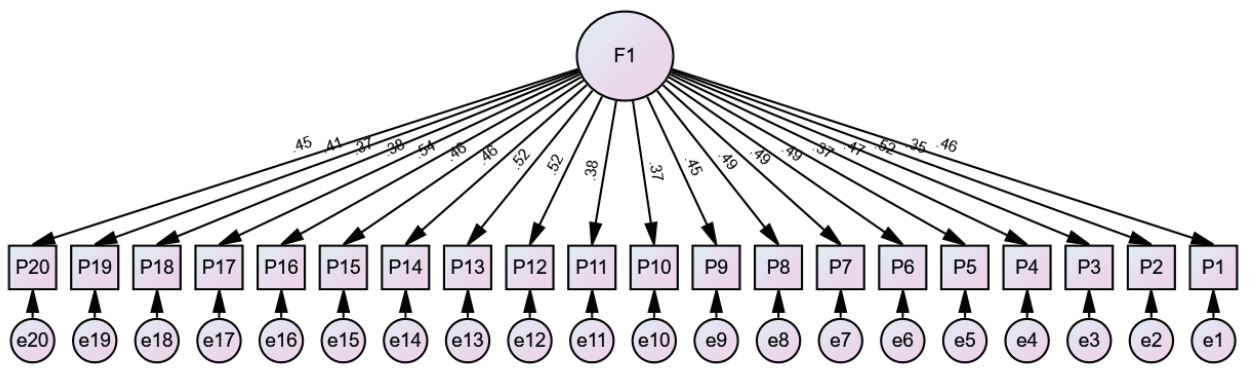

$\mathrm{X}^{2} / \mathrm{gl}=3.252 ; \mathrm{GFI}=0.923 ; \mathrm{CFI}=0.840 ; \mathrm{RMSEA}=0.056 ; \mathrm{SRMR}=0.0499$

Figura 2. Diagrama de senderos del factor Auto asertividad del Auto informe de conducta asertiva (ADCA - 1) 
En la figura 3 se indican los índices de ajuste de la propuesta original planteada por los autores del test de auto informe de conducta asertiva ADCA, específicamente para la dimensión heteroasertividad, $\mathrm{X}^{2} / \mathrm{gl}=3.908 ; \mathrm{GFI}=936 ; \mathrm{CFI}=0.853$; RMSEA $=0.063$; SRMR $=0.0517$, los índices de ajuste indican que el modelo propuesto no mide idóneamente la heteroasertividad, solo el GFI y el SRMR presenta índices adecuados. (Escobedo, Hernández, Estebané y Martínez, 2016).

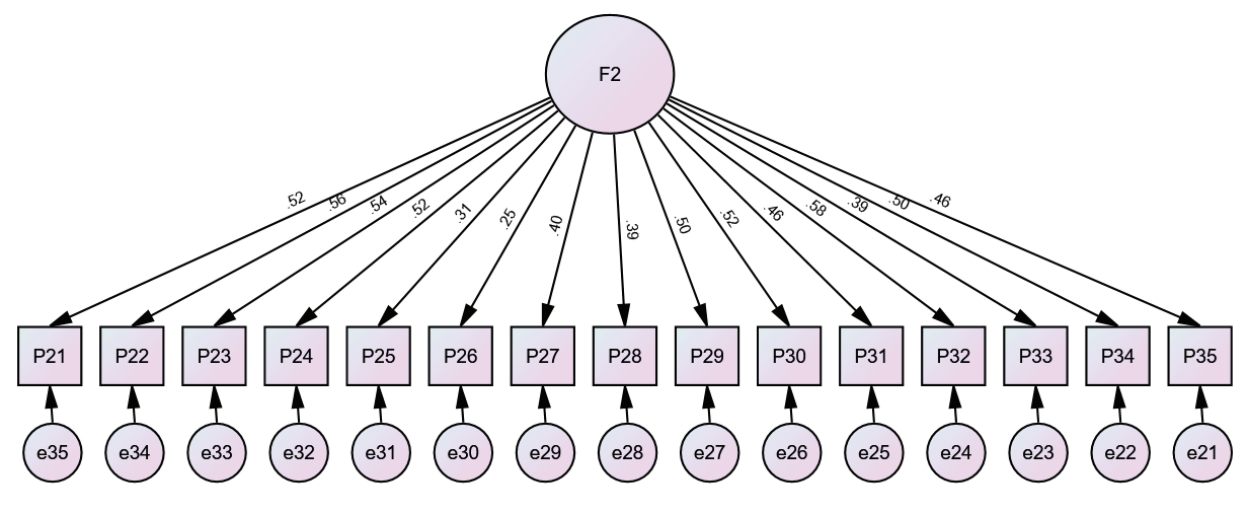

$\mathrm{X}^{2} / \mathrm{gl}=3.908 ; \mathrm{GFI}=0.936 ; \mathrm{CFI}=0.853 ; \mathrm{RMSEA}=0.063 ; \mathrm{SRMR}=0.0517$

Figura 3. Diagrama de senderos del factor Heteroasertividad del Auto informe de conducta asertiva (ADCA - 1)

\section{Análisis de ítems}

En la tabla 2, se observa el análisis descriptivo de los ítems correspondientes a la dimensión autoasertividad. A nivel general los ítems presentan adecuados valores en la asimetría y curtosis tal como lo mencionan Pérez y Medrano (2010) y Forero, Maydeu-Olivares, \& Gallardo-Pujol (2009) quienes indican que se encuentran entre +/- 1.5, a la vez los valores de los índices de homogeneidad corregido (IHC) son adecuados de acuerdo con Kline (2005) quien establece que el valor mínimo para que el ítem sea aceptable debe ser 0.30 , por último, los ítems presentan adecuadas comunalidades, exceptuando los ítems 9,11 y 19, los cuales no superan el 0.40 (Detrinidad, 2016).

En la tabla 3, se aprecia el análisis descriptivo de los ítems correspondientes a la dimensión heteroasertividad. A nivel general los ítems presentan adecuados valores en la asimetría y curtosis tal como lo mencionan Pérez y Medrano (2010) y Forero, Maydeu-Olivares, \& Gallardo-Pujol (2009) quienes indican que se encuentran entre $+/-1.5$, a la vez los valores de los índices de homogeneidad corregido (IHC) son adecuados de acuerdo con Kline (2005) quien establece que el valor mínimo para que el ítem sea aceptable debe ser 0.30, por último, los ítems presentan adecuadas comunalidades, exceptuando los ítems $21,24,28,33$ y 35 , los cuales no superan el 0.40 (Detrinidad , 2016). El ítem 26 se recomienda eliminar debido a que no cumple el criterio de Kline. 
Tabla 2

Análisis estadístico de los items de la dimensión autosertividad

\begin{tabular}{|c|c|c|c|c|c|c|c|}
\hline & Media & DS & Asimetría & Curtosis & IHC & $h^{2}$ & Condición \\
\hline P1 & 3,20 &, 841 & $-1,019$ & ,621 &, 420 &, 502 & \\
\hline P2 & 3,03 & 964 &,- 746 &,- 405 & ,324 & ,498 & \\
\hline P3 & 2,92 & ,983 &,- 642 &,- 571 &, 471 & ,449 & \\
\hline $\mathrm{P} 4$ & 2,91 & 1,005 &,- 612 &,- 690 &, 430 & ,445 & \\
\hline P5 & 2,87 & ,973 &,- 551 &,- 653 & ,338 &, 550 & \\
\hline P6 & 2,68 & 1,035 &,- 367 & $-1,009$ &, 431 &, 570 & \\
\hline $\mathrm{P} 7$ & 2,63 & 1,001 &,- 328 &,- 950 &, 436 &, 540 & \\
\hline P8 & 2,96 & ,913 &,- 599 &,- 430 &, 449 &, 487 & \\
\hline P9 & 3,01 & ,995 &,- 723 &,- 540 &, 416 & ,297 & $*$ \\
\hline $\mathrm{P} 10$ & 2,98 & ,977 &,- 700 &,- 490 & ,338 &, 433 & \\
\hline P11 & 2,85 & 1,064 &,- 529 &,- 953 &, 350 &, 393 & $*$ \\
\hline $\mathrm{P} 12$ & 2,68 & 1,000 &,- 365 &,- 913 &, 465 &, 593 & \\
\hline P13 & 3,00 & ,929 &,- 631 &,- 480 &, 483 & ,493 & \\
\hline $\mathrm{P} 14$ & 3,21 & ,979 & $-1,025$ &,- 081 &, 424 &, 479 & \\
\hline P15 & 3,10 & ,894 &,- 789 &,- 119 &, 419 & ,486 & \\
\hline P16 & 2,91 & ,947 &,- 625 &,- 462 &, 479 &, 556 & \\
\hline P17 & 3,10 & ,962 &,- 799 &,- 386 &, 354 & ,494 & \\
\hline P18 & 3,09 & 977 &,- 820 &,- 372 &, 342 &, 489 & \\
\hline P19 & 2,95 & 1,086 &,- 635 &,- 925 & ,379 &, 385 & * \\
\hline $\mathrm{P} 20$ & 2,87 & 979 &,- 652 &,- 518 &, 415 & ,446 & \\
\hline
\end{tabular}

* Eliminados

Tabla 3

Análisis estadístico de los ítems de la dimensión heteroasertividad

\begin{tabular}{|c|c|c|c|c|c|c|c|}
\hline & Media & DS & Asimetría & Curtosis & IHC & h2 & Condición \\
\hline P21 & 2,66 & ,985 &,- 343 &,- 883 &, 460 &, 383 & $*$ \\
\hline P22 & 2,79 & 1,014 &,- 451 &,- 880 & ,494 & 606 & \\
\hline $\mathrm{P} 23$ & 2,68 & 1,027 &,- 364 &,- 990 & ,468 & ,420 & \\
\hline $\mathrm{P} 24$ & 2,67 & 1,006 &,- 325 &,- 958 &, 462 & ,348 & $*$ \\
\hline P25 & 3,27 & ,908 & $-1,130$ & ,378 & ,303 & 607 & \\
\hline P26 & 3,27 & ,886 & $-1,139$ &, 554 &, 237 & ,641 & $*$ \\
\hline P27 & 2,65 & 1,003 &,- 303 &,- 965 & ,350 &, 542 & \\
\hline P28 & 3,05 & 965 &,- 777 &,- 375 & ,360 & ,240 & * \\
\hline P29 & 2,34 & 1,040 & ,047 & $-1,231$ & ,434 &, 410 & \\
\hline P30 & 2,58 & 1,036 &,- 210 & $-1,115$ &, 454 & ,404 & \\
\hline P31 & 2,71 & 1,055 &,- 393 & $-1,043$ &, 401 &, 471 & \\
\hline P32 & 2,61 & 1,003 &,- 294 &,- 982 &, 512 & ,401 & \\
\hline P33 & 2,82 & 1,101 &,- 519 & $-1,055$ & ,346 &, 219 & $*$ \\
\hline P34 & 2,53 & ,954 &,- 239 &,- 903 &, 450 & ,447 & \\
\hline P35 & 2,41 & 1,052 &,- 033 & $-1,236$ &, 415 & ,336 & * \\
\hline
\end{tabular}

\footnotetext{
* Eliminados
} 
En la tabla 4, se presentan los supuestos previos al análisis factorial exploratorio, se observa que existe una buena adecuación de los datos, debido a que el valor del KMO fue mayor a 0.80 lo cual es considerado adecuado según Ferrando y Anguino-Carrasco (2010). A la vez, el nivel de significancia prueba de esfericidad de Bartlett es de 0.000 ( $p<0.05)$, lo cual, según Montoya (2007) demuestra que no es una matriz de identidad, todo esto indica que es factible hacer el análisis factorial exploratorio.

\section{Tabla 4}

Supuestos previos AFE basados en 26 items

\begin{tabular}{lll}
\hline Prueba de KMO y Bartlett & & \\
\hline Medida Kaiser-Meyer-Olkin de adecuación de muestreo & & 0.897 \\
Prueba de esfericidad de Bartlett & Aprox. Chi-cuadrado & 3690.661 \\
& $\mathrm{gl}$ & 325 \\
& Sig. & 0.000 \\
\hline
\end{tabular}

En la tabla 5 se sugiere 6 factores teniendo en cuenta el criterio de Kaiser (1960) basado en el valor propio o "eigenvalue" mayor que uno, se encontró 47.807 $\%$ de varianza para los 6 factores extraídos, el cual puede ser considerado como de nivel adecuado de varianza tomando como referencia el $40 \%$, se considerado aceptable por Marín-García y Carneiro (2010). Sin embargo, el factor 6 solo tiene un ítem el cual es eliminado tal como se observa en la tabla 6, esto se basa en lo planteado por (Fabrigar et al., 1999; Ferrando y Anguiano-Carrasco, 2010) quienes indican que un factor debe tener entre 3 o 4 ítems. Eliminando este ítem y factor, la varianza con 5 factores es de $43.602 \%$.

Tabla 5

Varianza total explicada

\begin{tabular}{cccc}
\hline Factor & \multicolumn{3}{c}{ Autovalores iniciales } \\
& Total & \% de varianza & \% acumulado \\
\hline 1 & 5.789 & 22.266 & 22.266 \\
2 & 1.907 & 7.336 & 29.602 \\
3 & 1.290 & 4.963 & 34.565 \\
4 & 1.245 & 4.788 & 39.353 \\
5 & 1.105 & 4.249 & 43.602 \\
6 & 1.093 & 4.205 & 47.807 \\
\hline
\end{tabular}

En la tabla 6 se presentan la nueva agrupación en 5 factores de los 25 ítems después del análisis factorial exploratorio con rotación oblicua los factores fueron denominados de la siguiente manera: F1= Manejo a las críticas, F2= Manejo de 
respuestas negativas, $\mathrm{F} 3=$ Manejo del desconocimiento de un tema; F4= Manejo de las solicitudes de terceros y F5= Manejo de la percepción social

\section{Tabla 6}

Matriz de estructura con rotación oblicua

\begin{tabular}{|c|c|c|c|c|c|c|}
\hline & \multicolumn{6}{|c|}{ Factor } \\
\hline & 1 & 2 & 3 & 4 & 5 & 6 \\
\hline P22 & 0.582 & & & & & \\
\hline P15 & 0.532 & & & & & \\
\hline $\mathrm{P} 13$ & 0.524 & & & & & \\
\hline P8 & 0.495 & & & & & \\
\hline P31 & 0.488 & & & & & \\
\hline P5 & 0.479 & & & & & \\
\hline $\mathrm{P} 23$ & 0.462 & & & & & \\
\hline P10 & 0.382 & & & & & \\
\hline P34 & & 0.628 & & & & \\
\hline P29 & & 0.584 & & & & \\
\hline P32 & & 0.534 & & & & \\
\hline P30 & & 0.519 & & & & \\
\hline P27 & & 0.481 & & & & \\
\hline $\mathrm{P} 12$ & & & 0.673 & & & \\
\hline P16 & & & 0.617 & & & \\
\hline $\mathrm{P} 7$ & & & 0.479 & & & \\
\hline P17 & & & & 0.584 & & \\
\hline P18 & & & & 0.494 & & \\
\hline P25 & & & & 0.385 & & \\
\hline P20 & & & & 0.335 & & \\
\hline $\mathrm{P} 1$ & & & & & 0.593 & \\
\hline P3 & & & & & 0.562 & \\
\hline P14 & & & & & 0.493 & \\
\hline $\mathrm{P} 4$ & & & & & 0.416 & \\
\hline $\mathrm{P} 2$ & & & & & 0.323 & \\
\hline P6 & & & & & & $0.497 *$ \\
\hline
\end{tabular}

Método de extracción: cuadrados mínimos no ponderados.

Método de rotación: Oblimin con normalización Kaiser.

*Ítem eliminado 
En la figura 4 se presentan los índices de ajuste de la nueva propuesta del test de auto informe de conducta asertiva ADCA- 25 con 5 factores; los índices de ajuste son $\mathrm{X}^{2} / \mathrm{gl}=2.225$; $\mathrm{GFI}=0.936 ; \mathrm{CFI}=0.900 ; \mathrm{RMSEA}=0.041$; $\mathrm{SRMR}=0.0463$, los índices de ajuste indican que el modelo propuesto mide idóneamente la heteroasertividad, sólo el GFI y el SRMR presenta índices adecuados. (Escobedo, Hernández, Estebané y Martínez, 2016).

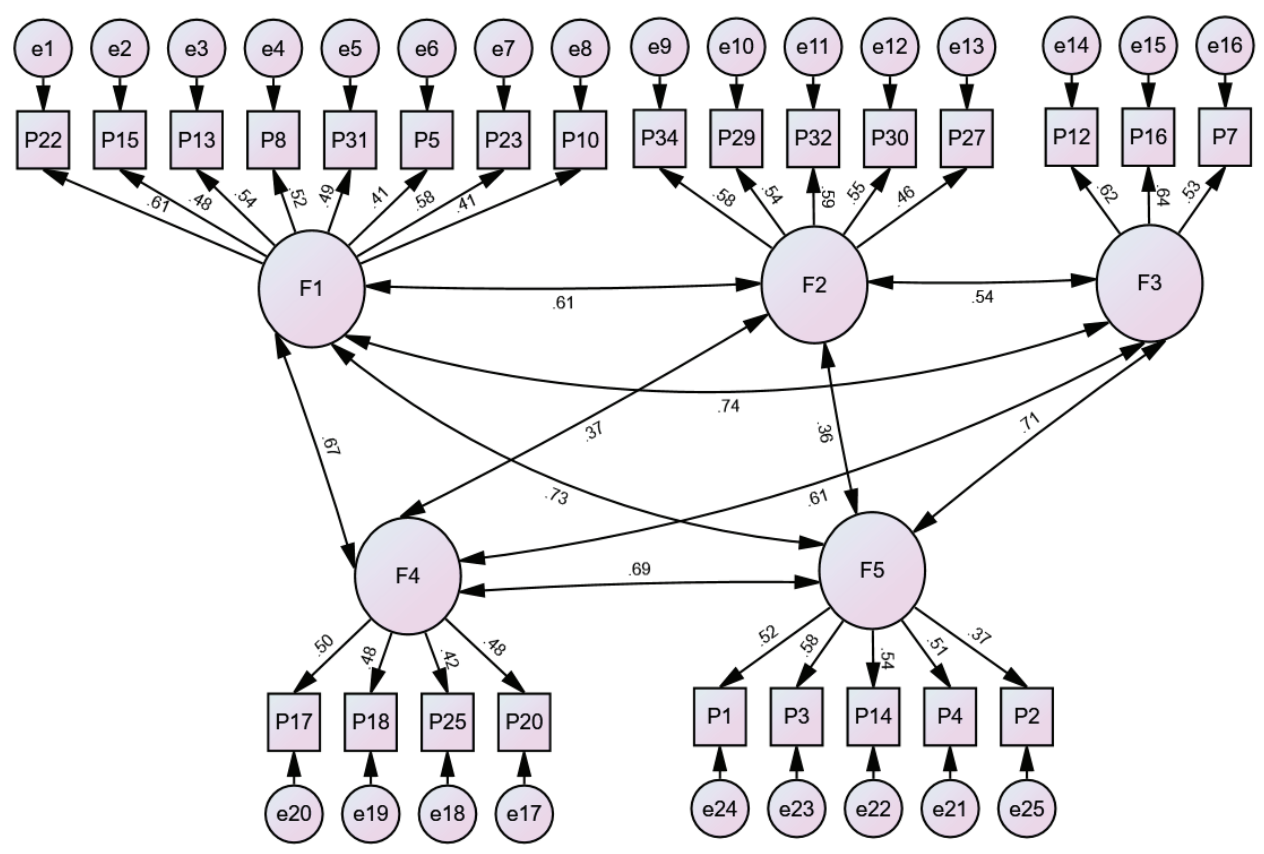

$\mathrm{X}^{2} / \mathrm{gl}=2,225 ; \mathrm{GFI}=0.936 ; \mathrm{CFI}=0.900 ; \mathrm{RMSEA}=0.041 ; \mathrm{SRMR}=0.0463$

Figura 4. Diagrama de senderos de la nueva propuesta del Auto informe de conducta asertiva (ADCA - 25)

En la tabla 7 se presentan la distribución final de los ítems en la propuesta final de 5 factores así mismo también se observa la numeración final.

En la figura 5 se presenta el diagrama de senderos y los índices del modelo bifactor de la propuesta de 5 factores propuesta en este trabajo, los cuales indican índices adecuados, además se postula que es viable de forma parcial obtener una puntuación global de la nueva propuesta, $\omega \mathrm{h}=0.784(\omega \mathrm{h}>0.70$ criterio óptimo); Hng $=0.851$ (Hng $>0.70$ criterio óptimo); $\mathrm{ECV}=0.588$ ( $\mathrm{ECV}>0.60$ criterio que indica poca varianza común); $\mathrm{PUC}=0.810$ ( $\mathrm{PUC}>0.70$ criterio óptimo) (Domínguez-Lara y Rodríguez, 2017). Sin embargo, esta debería ser puesta a prueba en posteriores investigaciones. 
Tabla 7

Distribución de la propuesta post análisis psicométrico

\begin{tabular}{|c|c|c|c|}
\hline Factores & $\begin{array}{c}\text { Numeración } \\
\text { original }\end{array}$ & $\begin{array}{l}\text { Numeración } \\
\text { final }\end{array}$ & Ítems \\
\hline \multirow{8}{*}{ F1 } & $\mathrm{P} 22$ & 1 & Me irrita mucho cuando me llevan la contraria. \\
\hline & $\mathrm{P} 15$ & 2 & Cuando creo haber cometido un error, busco excusas que me justifiquen. \\
\hline & $\mathrm{P} 13$ & 3 & $\begin{array}{l}\text { Me cuesta trabajo aceptar las críticas que hago, aunque comprenda que } \\
\text { son justas. }\end{array}$ \\
\hline & P8 & 4 & Me siento mal cuando tengo que cambiar alguna opinión. \\
\hline & P31 & 5 & Me disgusta que me critiquen. \\
\hline & P5 & 6 & Cuando me equivoco, me cuesta reconocerlo ante los demás. \\
\hline & $\mathrm{P} 23$ & 7 & Me molesta que los demás no comprendan mi razón o mis sentimientos. \\
\hline & $\mathrm{P} 10$ & 8 & Cuando pregunto algo que desconozco, procuro justificar mi ignorancia. \\
\hline \multirow{5}{*}{$\mathrm{F} 2$} & P34 & 9 & Me desagrada que no se dé a las cosas la importancia que tienen. \\
\hline & P29 & 10 & $\begin{array}{l}\text { Me siento mal cuando compruebo que una persona que aprecio tome una } \\
\text { decisión equivocada }\end{array}$ \\
\hline & P32 & 11 & $\begin{array}{l}\text { Siento malestar hacia la persona que me niega algo razonable que le pido } \\
\text { de buena manera. }\end{array}$ \\
\hline & $\mathrm{P} 30$ & 12 & Me altero cuando veo a alguien comportándose de manera indebidamente. \\
\hline & P27 & 13 & $\begin{array}{l}\text { Me desagrada comprobar que la gente no se esfuerce demasiado en hacer } \\
\text { su trabajo lo mejor posible. }\end{array}$ \\
\hline \multirow{3}{*}{ F3 } & $\mathrm{P} 12$ & 14 & $\begin{array}{l}\text { Me siento mal conmigo mismo/a, si no entiendo nada que algo me están } \\
\text { explicando. }\end{array}$ \\
\hline & P16 & 15 & Cuando descubro que no se algo, me siento mal conmigo mismo/a. \\
\hline & P7 & 16 & Me enfado, si no consigo hacer las cosas perfectamente. \\
\hline \multirow{4}{*}{ F4 } & $\mathrm{P} 20$ & 17 & Cuando me hacen elogios, me pongo nerviosa/o y no sé qué hacer. \\
\hline & $\mathrm{P} 25$ & 18 & Me molesta que me pidan ciertas cosas, aunque lo hagan con educación. \\
\hline & P18 & 19 & Me cuesta hacer favores. \\
\hline & P17 & 20 & Me cuesta hacer preguntas. \\
\hline \multirow{5}{*}{ F5 } & P4 & 21 & Me disgusta que los demás me vean nervioso. \\
\hline & $\mathrm{P} 14$ & 22 & Cuando me critican sin razón, me pone nervioso tener que defenderme \\
\hline & P3 & 23 & $\begin{array}{l}\text { Cuando hago algo que creo que no les gusta a los demás, siento miedo o } \\
\text { vergüenza de lo que puedan pensar de mí. }\end{array}$ \\
\hline & $\mathrm{P} 1$ & 24 & $\begin{array}{l}\text { Cuando alguien dice algo con lo que no estoy de acuerdo, me pone nervi- } \\
\text { oso tener que exponer mi propia opinión. }\end{array}$ \\
\hline & $\mathrm{P} 2$ & 25 & Cuando estoy enfadado me molesta que los demás se den cuenta. \\
\hline
\end{tabular}

Nota: F1= Manejo a las críticas, F2= Manejo de respuestas negativas, F3= Manejo del desconocimiento de un tema; F4= Manejo de las solicitudes de terceros, F5= Manejo de la percepción social 


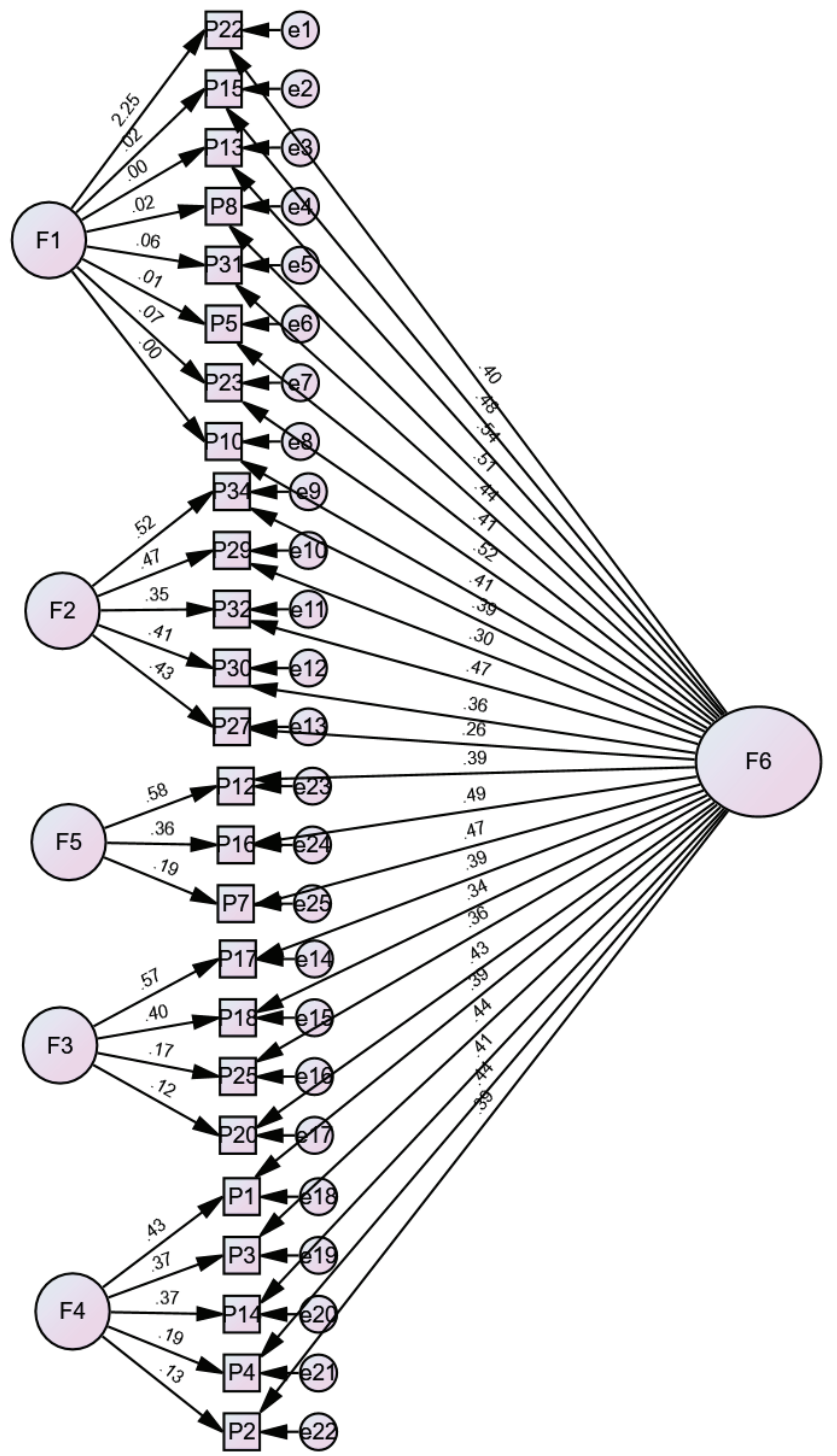

$\mathrm{X}^{2} / \mathrm{gl}=2.236 ; \mathrm{GFI}=0.940 ; \mathrm{CFI}=0.901 ; \mathrm{RMSEA}=0.041 ; \mathrm{SRMR}=0.0494 ; \omega_{\mathrm{h}}=0.784 ;$ $\mathrm{H}_{\mathrm{ng}}=0.851 ; \mathrm{ECV}=0.588 ; \mathrm{PUC}=0.810$

Figura 5. Propuesta exploratoria de un modelo bifactor para la distribución final post análisis psicométrico

\section{Confiabilidad}

En la tabla 8 se indican los valores de confiabilidad para los 5 factores y la escala general, A nivel general la escala obtiene un alfa ordinal de 0.902 y un coeficiente omega $\Omega=0.902$ que equivalen a una magnitud muy alta, para el caso de los 
factores 1, 2, 3 y 4 se ubican en una categoría alta, por último, el factor 4 se ubica en la categoría moderada (Ruiz Bolívar, 2002; Paella y Martins, 2003; Orozco, Labrador y Palencia, 2002)

Tabla 8

Confiabilidad con la propuesta de 5 factores

\begin{tabular}{ccc}
\hline Factor & Alfa ordinal & Omega $\mathbf{\Omega}$ \\
\hline F1 & 0.733 & 0.734 \\
F2 & 0.676 & 0.678 \\
F3 & 0.625 & 0.627 \\
F4 & 0.530 & 0.531 \\
F5 & 0.628 & 0.631 \\
ESCALA & 0.902 & 0.902 \\
\hline
\end{tabular}

En la tabla 9 se presenta el estadístico U de Mann-Whitney para verificar si existen diferencias por sexo en los factores, se presentan diferencias en los factores 2 y 3 , por lo tanto, se elaboraron percentiles para el sexo femenino y masculino.

Tabla 9

Estadísticos de prueba

\begin{tabular}{lcccccc}
\hline & F1 & F2 & F3 & F4 & F5 & ASERTIVIDAD \\
\hline U de Mann-Whitney & 62326.5 & 55712.5 & 59436.5 & 63496.0 & 61673.0 & 65320.5 \\
Sig. & 0.179 & 0.000 & 0.017 & 0.349 & 0.114 & 0.775 \\
\hline
\end{tabular}

a. Variable de agrupación: SEXO

\section{Percentiles}

En la tabla 10 se aprecian los percentiles de los 5 factores y de la escala general con 25 ítems, se observa que en el factor 2 y 3 se muestran puntajes para el sexo femenino y masculino.

\section{Tabla 10}

Percentiles de la nueva propuesta

\begin{tabular}{|c|c|c|c|c|c|c|c|c|c|}
\hline \multirow{3}{*}{ Percentil } & \multirow{3}{*}{$\begin{array}{c}\text { F1 } \\
\begin{array}{l}\text { Puntajes } \\
\text { directos }\end{array}\end{array}$} & \multicolumn{2}{|c|}{ F2 } & \multicolumn{2}{|c|}{ F3 } & F4 & F5 & ASERTIVIDAD & \multirow{3}{*}{ Categoría } \\
\hline & & & Puntaje & directos & & & \multirow{2}{*}{\multicolumn{2}{|c|}{ Puntajes directos }} & \\
\hline & & Femenino & Masculino & Femenino & Masculino & & & & \\
\hline 1 & 12 & 5 & 5 & 3 & 3 & 6 & 6 & 39 & \multirow{3}{*}{ Muy bajo } \\
\hline 10 & 17 & 8 & 9 & 5 & 5 & 9 & 11 & 57 & \\
\hline 20 & 19 & 9 & 11 & 6 & 7 & 9 & 13 & 62 & \\
\hline 30 & 20 & 10 & 12 & 7 & 7 & 10 & 14 & 66 & \multirow{2}{*}{ Bajo } \\
\hline 40 & 22 & 11 & 12 & 8 & 8 & 11 & 15 & 69 & \\
\hline 50 & 23 & 12 & 13 & 8 & 9 & 12 & 16 & 72 & \multirow{2}{*}{ Medio } \\
\hline 60 & 25 & 13 & 14 & 9 & 9 & 13 & 17 & 75 & \\
\hline 70 & 26 & 14 & 15 & 9 & 10 & 14 & 18 & 78 & \multirow{2}{*}{ Alto } \\
\hline 80 & 28 & 15 & 16 & 10 & 11 & 15 & 19 & 82 & \\
\hline 90 & 29 & 17 & 17 & 11 & 12 & 16 & 20 & 86 & \multirow{2}{*}{ Muy alto } \\
\hline 99 & 32 & 19 & 20 & 12 & 13 & 17 & 21 & 94 & \\
\hline
\end{tabular}




\section{DISCUSIÓN}

La investigación presente de tipo instrumental se aúna a los trabajos de investigación que estudian las propiedades psicométricas de los instrumentos, de manera específica se analizó las evidencias de validez basada en la estructura interna, la confiabilidad y se plantea una nueva propuesta de 25 ítems.

El análisis factorial confirmatorio de la propuesta inicial del ADCA-1 indica que los índices de ajuste no son óptimos $\mathrm{X}^{2} / \mathrm{gl}=2.648$; $\mathrm{GFI}=0.883 ; \mathrm{CFI}=0.807$; $\mathrm{RMSEA}=0.048$; $\mathrm{SRMR}=0.0532$ tomando como referencia los criterios de Escobedo, Hernández, Estebané y Martínez (2016) y Wheaton, Muthen, Alwin, \& Summers (1977). No se encontraron autores que trabajaron el análisis factorial confirmatorio; estos resultados presentan evidencias distintas a las mencionadas por García y Magaz (2000) quienes afirman que el instrumento tiene una adecuada estructura interna y evidencias de validez.

Es importante indicar que sólo elaboraron la validez de contenido, lo cual hace discutible su uso con la estructura original en estudiantes peruanos, a pesar que García (2014) menciona que la escala posee un alfa de Cronbach de 0.773 y 0.752 para la autoasertividad y heteroasertividad respectivamente. Por su parte, Becerra, Vásquez, López, Becerra, Cerna y Medina (2012) consiguieron un coeficiente de correlación de 0.73 entre las dimensiones autoasertividad y heterosasertividad, estos resultados no están acorde a lo encontrado en esta investigación debido a que de los 35 ítems iniciales solo cumplen con los criterios idóneos de asimetría, curtosis, índices de homogeneidad corregido y comunalidad de 26 ítems, según Pérez y Medrano (2010) y Forero, Maydeu-Olivares \& Gallardo-Pujol (2009), Kline (2005) y (Detrinidad, 2016). De estos para el análisis final quedaron 25 ítems debido a que un factor cargaba con un solo ítem y como mínimo deben tener 3 o 4 ítems (Ferrando y Anguiano-Carrasco, 2010); a nivel general, los análisis evidencian que la estructura después de la revisión plantea la existencia de 4 factores y no los dos iniciales planteados en la propuesta inicial, a la vez, no se pueda afirmar de forma categórica la existencia de un factor general debido a que los valores del análisis de un modelo bifactor no son del todo óptimos, $\omega \mathrm{h}=0.784$ ( $\omega \mathrm{h}>0.70$ criterio óptimo); Hng $=0.851$ (Hng $>0.70$ criterio óptimo); $\mathrm{ECV}=0.588$ (ECV $>0.60$ criterio que indica poca varianza común); $\mathrm{PUC}=0.810$ ( $\mathrm{PUC}>0.70$ criterio óptimo) (Domínguez-Lara y Rodríguez, 2017).

Los hallazgos del presente estudio presentan una propuesta de evaluación de la asertividad que tiene 5 factores definidos, se recomienda explorar el funcionamiento de la propuesta con otras variables, poner a prueba la estructura interna a través de análisis confirmatorios, evaluar la invariancia métrica y explorar la fiabilidad con métodos distintos como el de test-retest, para evaluar la estabilidad en el tiempo de la propuesta. 


\section{CONCLUSIONES}

1. El Autoinforme de conducta asertiva (ADCA-1) en su propuesta original no presente evidencias de validez en estudiantes peruanos

2. El modelo de 5 factores propuesto explica el $43.602 \%$ de la varianza del constructo.

3. El modelo de 5 factores propuesto presenta adecuados índices de ajuste lo que indica que hay evidencias de validez de constructo.

4. El modelo propuesto de Autoinforme de conducta asertiva es altamente confiable.

\section{Agradecimientos / Acknowledgments:}

Los autores agradecen el apoyo brindado por los directores de las instituciones educativas quienes autorizaron la realización del presente estudio.

\section{Fuentes de financiamiento / Funding:}

Autofinanciado

\section{Rol de los autores / Authors Roles:}

FJRQ: analizó los resultados estadísticos e interpretación.

CRMT: analizó los resultados estadísticos e interpretación.

EREA: recogió y proceso la data.

MAMC: recogió la data.

AGM: redacción, interpretación de resultados.

JWPC: redacción, interpretación de resultados y corrección.

\section{Aspectos éticos / legales; Ethics / legals:}

Los autores declaran haber respetado lo establecido por las normativas éticas que regulan el ejercicio profesional (Código de Ética del Colegio de Psicólogos del Perú). Se protegió la confidencialidad de la información personal e institucional, asegurando el anonimato de las personas e instituciones involucradas en la muestra.

\section{Conflicto de intereses / Competing interests:}

Los autores declaran bajo juramento no haber incurrido en conflicto de interés al realizar este artículo. 


\section{REFERENCIAS}

Abad, A.; Servín, L. A. (1984). Introducción al muestreo. México: Limusa.

Aguilarte, I.; Calcurián, I. y Ramírez, Y. (2010). La comunicación asertiva para mejorar las relaciones interpersonales en los docentes. Tesis en maestría. Facultad de Humanidades y Educación. Universidad central de Venezuela. Barcelona.

Ayvar, H. (2016). La autoestima y la asertividad en adolescentes de educación secundaria de un colegio estatal y particular de un sector del distrito de Santa Anita. Avances en Psicología - UNIFE. 24 (2) pp. 193-203. DOI: https://doi.org/10.33539/avpsicol.2016. v24n2.154

Ayvar, H. (2016). La autoestima y la asertividad en adolescentes de educación secundaria de un colegio estatal y particular de un sector del distrito de Santa Anita. Revista de Psicología. Universidad Femenina del Sagrado Corazón. Lima. Perú. Recuperado de http://www.unife.edu.pe/publicaciones/revistas/psicologia/2016_2/193.pdf

Becerra, S.; Vásquez, J.; López, D.; Becerra, S., Cerna, J. y Medina, J. (2012). Asertividad en escolares de Lima Metropolitana. Disponible en: http://www.unfv.edu.pe/facultades/faps/images/pdf/2012/Unidad_Investigacion/Resumen2012_02.pdf

Detrinidad, E. (2016). Análisis Factorial Exploratorio y Confirmatorio aplicado al modelo de secularización propuesto por Inglehart-Norris. Periodo 2010-2014 (Estudio de caso España, Estados Unidos, Alemania, Holanda) WSV. (Tesis de Maestría, Universidad De Granada).

Escobedo, M., Hernández, J., Estebané, V. y Martínez, G. (2016). Modelos de Ecuaciones Estructurales: Características, Fases, Construcción, Aplicación y Resultados. Revista Ciencia \& Trabajo, 18 (55), 16-22. http://dx.doi.org/10.4067/S071824492016000100004

Fabrigar, L. R., Wegener, D. T., MacCallum, R. C., y Strahan, E. J. (1999). Evaluating the use of exploratory factor analysis in psychological research. Psychological Methods, 4(3), 272-299.

Ferrando, P. y Anguino-Carrasco, C. (2010). Análisis factorial como técnica de investigación psicológica. Papeles del Psicólogo. 31 (1), 18-33 Disponible en: http://www. papelesdelpsicologo.es/pdf/1793.pdf; DOI: 2006-07-04

Forero, C. G., Maydeu-Olivares, A., \& Gallardo-Pujol, D. (2009). Factor analysis with ordinal indicators: A Monte Carlo study comparing DWLS and ULS estimation. Structural Equation Modeling, 16(4), 625-641. https://doi.org/10.1080/10705510903203573

García, V. (2014). Propiedades psicométricas del Auto informe de conducta asertiva en adolescentes de educación secundaria. Revista de investigación de estudiantes de Psicología JANG. 3(1) pp. 34-57. DOI: https://doi.org/10.18050/jang.v8i2

García, M. y Magaz, A. (1995). Ratones, Dragones y Seres Humanos Auténticos. Madrid: Ed. CEPE.

García, M. y Magaz, A. (2000). Escala de evaluación de la asertividad ADCA-1. Manual técnico. Madrid: CEPE, 2000. 
Grajeda, A. (2018). Construcción y validación de un test de aptitud funcional selectora visual en estudiantes de escuelas estatales del primer grado de primaria del distrito de Jesús María, 2018. Tesis Doctoral. Universidad César Vallejo. Recuperado de http://repositorio.ucv.edu.pe/bitstream/handle/UCV/21249/Grajeda_MAT. pdf?sequence $=1 \&$ isAllowed $=y$

Hernández, R., Fernández, C. y Baptista, P. (2010). Metodología de la Investigación (5ed.). México: McGraw-Hill Interamericana.

Inglés, C. Méndez, F. y Hidalgo, M. (2000). Cuestionario de evaluación de dificultades interpersonales en la adolescencia. Psicothema. 12 (3) pp. 390-398. Disponible en: http://www.psicothema.com/pdf/347.pdf

Kaiser, H.F. (1960). The application of electronic computers to factor analysis. Educational and Psychological Measurement, 20, 141-151 https://doi. org/10.1177/001316446002000116

León, M. (2009). Revisión de la escala de asertividad de Rathus adaptada por León y Vargas - 2009. Revista Reflexiones. 93 (1) pp. 157-171. Disponible en: https://revistas. ucr.ac.cr/index.php/reflexiones/article/view/13751

Loyola, P. (2012). Propiedades Psicométricas del Autoinforme de Conducta Asertiva ADCA - 1 en Adolescentes del Distrito de Huamachuco (Tesis de licenciatura). Universidad César Vallejo, Trujillo, Perú.

Ministerio de Educación del Perú (2019). Estadística de la calidad educativa. Lima: MINEDU. Recuperado de http://escale.minedu.gob.pe/padron-de-iiee

Montero, I. y León, O. (2002). Clasificación y descripción de las metodologías de investigación en Psicología. Revista Internacional de Psicología Clínica y de la Salud. 2(3), 503-508. Disponible en: https://aepc.es/ijchp/articulos_pdf/ijchp-53.pdf

Montoya, O. (Mayo/julio, 2007). Aplicación del análisis factorial a la investigación de mercados. Caso de estudio. Scientia et Technica, 13(35), 281-286. DOI: http://dx.doi. org/10.22517/23447214.5443

Morán, V. y Olaz, F. (2014). Instrumentos de evaluación de habilidades sociales en América Latina: un análisis bibliométrico. Revista de Psicología - Universidad de Chile. 23 (1) pp. 93-105. DOI: 10.5354/0719-0581.2014.32877

Nunnally, J. (1978). Psychometric Theory. McGraw-Hill. New York.

Ñaupas, H., Mejía, E., Novoa, E. y Villagomez, A. (2011). Metodología de la investigación cientifica y asesoramiento de tesis. Lima: Editorial Humberto Naupas Paitan.

Palella, S. y Martins, F. (2003). Metodología de la Investigación cuantitativa. Caracas: Fedupel.

Pérez, E. y Medrano, L. (2010). Análisis Factorial Exploratorio: Bases Conceptuales y Metodológicas. Revista Argentina de Ciencias del Comportamiento. 2 (1), 58-66. DOI: https://doi.org/10.32348/1852.4206.v2.n1.15924

Ruiz Bolívar, C. (2002). Instrumentos de investigación educativa. Venezuela: Fedupel 
Orozco, C., Labrador, M. y Palencia, A. (2002). Metodología. Manual teórico práctico de metodología para tesistas, asesores, tutores y jurados de trabajos de investigación y ascenso. Venezuela: Ofimax de Venezuela

Quian, M. y Santos, J. (2015). Análisis de Ítems con jMetrik. Guatemala: Dirección General de evaluación e investigación educativa. Ministerio de educación. Disponible en: https://issuu.com/digeduca/docs/analisis_items_jmetrik

Kline, P. (2005). Principles and practice of structural equation modelling. (2nd Edition ed.). New York: The Guilford Press

Roca, R. (2015). Know Mads. Los trabajadores del futuro. Madrid: LID Editorial.

Ruiz Bolívar, C. (2002). Instrumentos de investigación educativa. Caracas: Fedeupel.

Wheaton, B., Muthen, B., Alwin, D., F., \& Summers, G. (1977). Assessing Reliability and Stability in Panel Models, Sociological Methodology, 8(1), 84-136. Doi: http://dx.doi. org/10.2307/270754 


\section{ANEXO}

\section{ADCA- 25 (Propuesta exploratoria para Perú)}

\section{Rosario, Manrique, Estrada, Misari, Grajeda y Pomahuacre (2020)}

Nombres y apellidos:

Edad:

Sexo:

Fecha:

Para responder satisfactoriamente al presente instrumento, marque con una $\mathrm{X}$ la columna que indique de forma precisa tu reacción en cada situación, Tener en cuenta que no existen respuestas correctas o incorrectas.

\begin{tabular}{|c|c|c|c|c|c|}
\hline $\mathrm{N}^{\circ}$ & Ítems & Nunca & $\begin{array}{c}\text { A } \\
\text { veces }\end{array}$ & $\begin{array}{c}\text { A } \\
\text { menudo }\end{array}$ & Siempre \\
\hline 1 & Me irrita mucho cuando me llevan la contraria. & & & & \\
\hline 2 & Cuando creo haber cometido un error, busco excusas que me justifiquen. & & & & \\
\hline 3 & Me cuesta trabajo aceptar las críticas que hago, aunque comprenda que son justas. & & & & \\
\hline 4 & Me siento mal cuando tengo que cambiar alguna opinión & & & & \\
\hline 5 & Me disgusta que me critiquen. & & & & \\
\hline 6 & Cuando me equivoco, me cuesta reconocerlo ante los demás. & & & & \\
\hline 7 & Me molesta que los demás no comprendan mi razón o mis sentimientos. & & & & \\
\hline 8 & Cuando pregunto algo que desconozco, procuro justificar mi ignorancia. & & & & \\
\hline 9 & Me desagrada que no se dé a las cosas la importancia que tienen. & & & & \\
\hline 10 & $\begin{array}{l}\text { Me siento mal cuando compruebo que una persona que aprecio tome una decisión } \\
\text { equivocada }\end{array}$ & & & & \\
\hline 11 & $\begin{array}{l}\text { Siento malestar hacia la persona que me niega algo razonable que le pido de buena } \\
\text { manera. }\end{array}$ & & & & \\
\hline 12 & Me altero cuando veo a alguien comportándose de manera indebidamente. & & & & \\
\hline 13 & $\begin{array}{l}\text { Me desagrada comprobar que la gente no se esfuerce demasiado en hacer su trabajo } \\
\text { lo mejor posible. }\end{array}$ & & & & \\
\hline 14 & Me siento mal conmigo misma, si no entiendo nada que algo me están explicando & & & & \\
\hline 15 & Cuando descubro que no se algo, me siento mal conmigo mismo/a. & & & & \\
\hline 16 & Me enfado, si no consigo hacer las cosas perfectamente & & & & \\
\hline 17 & Cuando me hacen elogios, me pongo nerviosa/o y no sé qué hacer. & & & & \\
\hline 18 & Me molesta que me pidan ciertas cosas, aunque lo hagan con educación. & & & & \\
\hline 19 & Me cuesta hacer favores. & & & & \\
\hline 20 & Me cuesta hacer preguntas. & & & & \\
\hline 21 & Me disgusta que los demás me vean nervioso. & & & & \\
\hline 22 & Cuando me critican sin razón, me pone nervioso tener que defenderme & & & & \\
\hline 23 & $\begin{array}{l}\text { Cuando hago algo que creo que no les gusta a los demás, siento miedo o vergüenza } \\
\text { de lo que puedan pensar de mí. }\end{array}$ & & & & \\
\hline 24 & $\begin{array}{l}\text { Cuando alguien dice algo con lo que no estoy de acuerdo, me pone nervioso tener } \\
\text { que exponer mi propia opinión. }\end{array}$ & & & & \\
\hline 25 & Cuando estoy enfadado me molesta que los demás se den cuenta. & & & & \\
\hline
\end{tabular}

Muchas gracias. 